Doug Geisler, Eva K. Grebel, and Dante Minniti, eds.

\title{
Cluster Distances using $\delta$ Sct Stars; Oscillating Blue Stragglers in $\omega$ Cen
}

\section{L.M. Freyhammer}

Royal Observatory of Belgium, Ringlaan 3, 1180 Brussels, Belgium and University of Brussels (VUB), Pleinlaan 2, 1050 Brussels, Belgium

\author{
Jørgen Otzen Petersen
}

Niels Bohr Institute for Astronomy, Physics and Geophysics; Astronomical Observatory, Juliane Maries Vej 30, DK-2100 Copenhagen $\varnothing$, Denmark

\begin{abstract}
The complicated star formation history of the most massive globular cluster in our Galaxy - $\omega$ Centauri - is seen as a presence of an asymmetric spatial distribution of two stellar populations having different ages and metallicities. The cluster hosts the largest known sample of Population II $\delta$ Sct variables, or SX Phœnicis stars, which are valuable Galactic and extragalactic distance estimators. We discuss the applicability of these variables for estimating distances to the cluster, and compare different techniques for the critical identifications of oscillation modes.
\end{abstract}

\section{Introduction}

Pulsating variables are good distance estimators, as demonstrated among others by Walker et al. (2001) for the Magellanic Clouds, McNamara (2000a) for the Carina galaxy and $\omega$ Cen, and McNamara et al. (2000b) for the Galactic bulge. The class of SX Phe stars seems to be very precise for this purpose, as predicted from theoretical (e.g. Petersen \& Christensen-Dalsgaard 1996, 1999) and empirical investigations. Furthermore, when including colour terms in periodluminosity (PL) relations, the scatter in the derived absolute magnitudes reduces significantly. For Pop. I $\delta$ Sct stars Petersen \& Christensen-Dalsgaard (1999) determined the following theoretical PLCZ-relation:

$$
M_{\text {bol }}=-3.785 \log \Pi_{0}-12.179 \log \mathrm{T}_{\mathrm{e}}-0.277[\mathrm{Fe} / \mathrm{H}]+44.837
$$

with an intrinsic scatter of $\pm 00^{\mathrm{m}} 026 . M_{\mathrm{bol}}$ is the bolometric magnitude and $\Pi_{0}$ is the period of the fundamental radial mode. Similarly, by taking into account smaller periods, lower $[\mathrm{Fe} / \mathrm{H}]$ and higher $\mathrm{T}_{\mathrm{e}}$, we derive for the Pop. II stars:

$$
M_{\text {bol }}=-3.765 \log \Pi_{0}-12.224 \log \mathrm{T}_{\mathrm{e}}-0.114[\mathrm{Fe} / \mathrm{H}]+45.205
$$

with an intrinsic scatter of $\pm 00^{\mathrm{m}} 021$. 


\section{Period-Luminosity relations and pulsation modes}

The period $\Pi_{0}$ in Eqs. 1 and 2 may be obtained by (i) using theoretical period ratios to identify modes for stars oscillating in two radial modes, or (ii) alternatively, one may locate linear sequences in PL-diagrams for several mono-periodic variables and identify the modes from period ratios between such sequences or from single stars with known modes. For SX Phe stars, corrections for metallicity and temperature (PLZC-relations) are necessary. McNamara (2000a, 2000b) identified modes for 32 SX Phe stars in $\omega$ Cen with the 'diagram-sequence' method (ii) using additional criteria like a subjective "asymmetry index". $\mathrm{He}$ derived a true distance modulus of 14.0 for the cluster.

To verify McNamara's (2000a) distance modulus and check his mode identifications, we combined the modes with $V-I$ colours (Kaluzny et al. 1996, 1997) and own $B-V$ colours in Eq. 2. Based on standard evolutionary tracks, the surface gravity was fixed for all stars to $\log g=4.15 \pm 0.15$. Most stars in $\omega$ Cen have metallicities of -2.0 to $-1.4 \mathrm{dex}$ (Hilker et al. 2000), so we fixed $[\mathrm{Fe} / \mathrm{H}]$ to $-1.7 \mathrm{dex}$, corresponding to $0 .{ }^{\mathrm{m}} 07$ uncertainty in the absolute magnitude. We derived temperatures with simple colour-temperature transformations, which contributes with errors of $0{ }^{\mathrm{m}} 06 . B-V$ temperatures are more abundance sensitive than $V-I$ and therefore less suited for our assumed metallicity. $B-V$ colours were only available for 10 stars, for which we identified modes by shifting the HIPPARCOS PL-relation by Petersen \& Dalsgaard (1999, their Eq. 4) to locations of two doublemode stars (Petersen et al. 2000).

In general, we confirm McNamara's (2000a) mode identifications. We find the median distance moduli: $(m-M)_{0}=13^{\mathrm{m}} 75 \pm 0.18 \mathrm{mag}$ and $13.7 \pm 0.3 \mathrm{mag}$ for $V-I$ and $B-V$ colours respectively. The errors are considerable but do not exclude McNamara's value. The available photometry of the variables does not provide mean colors and abundance estimates accurate enough to include colour terms in the PLRs. The most precise distance modulus for $\omega$ Cen, based on PLRs, is thus McNamara's (2000a) value.

Acknowledgments. We thank for support from IUAP P4/05 financed by the Belgian DWTC/SSTC, and from the Flemish Ministry for Foreign Policy, European Affairs, Science and Technology, under contract BIL 99/2.

\section{References}

Hilker, M. \& Richtler T. 2000, A\&A, 362, 895

Kaluzny, J., Kubiak, M., Szymański, M. et al. 1996, A\&AS, 120, 139

Kaluzny, J., Kubiak, M., Szymański, M. et al. 1997, A\&AS, 122, 471

McNamara, D.H. 2000a, PASP, 112, 1096

McNamara, D.H., Madsen, J.B., Barnes, J. 2000b, PASP, 112, 202

Petersen, J.O. \& Christensen-Dalsgaard, J. 1996, A\&A, 312, 463

Petersen, J.O. \& Christensen-Dalsgaard, J. 1999, A\&A, 352, 547

Petersen, J.O., Quaade, M., Freyhammer, L.M. \& Andersen, M.I. 2000, ASP Conf. Ser. Vol. 210, (San Francisco: ASP), 391

Walker, A.R. 2002, these proceedings 